\section{RECENT ADVANCES IN RADIO-ACTIVITX."}

IN 1904 I had the honour of giving an address at the Roval Institution on the subject of radio-activity. In the interval steady and rapid progress has been made in unravelling the tangled skein of radio-active phenomena. In the present lecture I shall endeavour to review very shortly some of the more important advances made in the last few years, but as I cannot hope to mention, even briefly, the whole additions to our knowledge in the various branches of the subject, I shall confine my attention to a few of the more salient facts in the development of which I have taken some small share.

In my previous lecture I based the explanation of radioactive phenomena on the disintegration theory put forward in 1903 by Rutherford and Soddy, which supposes that the atoms of the radio-active bodies are unstable systems which break up with explosive violence. This theory has stood the test of time, and has been invaluable in guiding the experimenter through the maze of radio-active complications. In its simplest form, the theory supposes that every second a certain fraction (usually very small) of the atoms present become unstable and explode with great violence, expelling in many cases a small portion of the disrupted atom at a high speed. The residue of the atom forms a new atomic system of less atomic weight, and possessing physical and chemical properties which markedly distinguish it from the parent atom. The atoms composing the new substance formed by the disintegration of the parent matter are also unstable, and break up in turn. The process of degradation of the atom, once started, proceeds through a number of distinct stages. These new products formed by the successive disintegrations of the parent matter are in most cases present in such extremely minute quantity that they cannot be investigated by ordinary chemical methods. The radiations from these substances, however, afford a very delicate method of qualitative and quantitative analysis, so that we can obtain some idea of the physical and chemical properties of substances existing in an amount which is far below the limit of detection of the balance or spectroscope.

The law that governs the breaking up of atoms is very simple and universal in its application. For any simple substance, the average number of atoms breaking up per second is proportional at any time to the number present. In consequence, the amount of radio-active matter decreases in a geometrical progression with the time. The "period" of any radio-active product, i.e. the time for half the matter to be transformed, is a definite and characteristic property of the product which is uninfluenced by any of the laboratory agents at our command. In fact, the period of any radio-active product, for example, the radium emanation, if determined with sufficient accuracy, might well be taken as a definite standard of time, independent of all terrestrial influences.

The law of radio-active transformation can be very simply and aptly illustrated by an hydraulic analogy. Suppose we take a vertical cylinder filled with water, with an opening near the base through which the water escapes through a high resistance. ${ }^{2}$ When the discharge is started the amount of water escaping per second is proportional to the height of water above the zero level of the cylinder. The height of water decreases in a geometrical progression with the time in exactly the same way as the amount of radio-active matter decreases. We can consequently take the height of the column of water as representing the amount of radio-active matter $A$ present at any time. The quantity of water escaping per second is a measure of the rate of disintegration of $A$ and also of the amount of the new substance $B$ formed per second by the disintegration of A. The "period" of the substance is controlled by the amount of resistance in the discharge circuit. A high resistance gives a small flow of water and a long period of transformation, and vice versa. By a suitable arrangement we can readily trace out the decay curve for such a case. A cork carrying a light vertical glass rod is floated on the water in the cylinder. A light camel's hair brush

${ }^{1}$ A discourse delivered at the Royal Institution on Friday, January $3 \mathrm{r}$, by Prof.

2 A short glass tube in which is placed a plug of glass wool is very suitable.

NO. 2OOI, VOL. 77] is attached at right angles, and moves over the surface of a smoked-glass plate. A vertical line drawn on the glass through the point of contact of the brush gives the axis of ordinates, while a horizontal line drawn through the brush when the water has reached its lowest level gives the axis of abscissæ. If the glass plate is moved with uniform velocity from the moment of starting the discharge a curve is traced on the glass which is identical in shape with the curve of decay of a radio-active product, where the ordinates at any time represent the relative amount of active matter present, and the abscissæ time. With such an apparatus we can illustrate in a simple way the increase with time of radio-active matter $B$, which is supplied by the transformation of a substance A. This will correspond, for example, to the growth of the radium emanation with time in a quantity of radium initially freed from emanation. Let us for convenience suppose that A has a much longer period than B. In the hydraulic analogy $A$ is represented by a high head of water discharging at its base through a circuit of high resistance into the top of another cylinder representing the matter $B$. The water from the cylinder B escapes at its base through a lower resistance. Suppose that initially only $A$ is present. In this case the water in the cylinder B stands at zero level. On opening the stop-cock connecting with $\mathrm{A}$, water flows into $B$. The rise of water with time in the cylinder $B$ is traced out in the same way as before by moving the glass plate at a constant rate across the tracing brush. If the period of $A$ is very long compared with that of $B$ the water is supplied to $B$ at a constant rate, and the water in $B$ reaches a constant maximum height when the rate of supply to $B$ equals the rate of escape from the latter. The curve traced out in that case is identical in shape with the " recovery curve" of a radio-active product supplied at a nearly constant rate. The quantity of matter reaches a maximum when the rate of supply equals its own rate of transformation. The relative height of the columns of water in $\mathrm{A}$ and $\mathrm{B}$ represents at any time the relative amounts of these substances present.

If the period is comparable with that of $B$, the height of water in B after reaching a maximum falls again, since as the height of $\mathrm{A}$ diminishes the supply to $\mathrm{B}$ decreases. Ultimately, the height of $\mathrm{B}$ will decrease in a geometrical progression with the time at a rate corresponding to the longer period of the two. This is an exact illustration of the way the amount of a radio-active substance $B$ varies when initially only the parent substance $\mathrm{A}$ is present. By using a number of cylinders in series, each with a suitable resistance, we can in a similar way illustrate in a quantitative manner the variation in amount with time of a number of products arising from successive disintegrations of a primary substance. By suitably adjusting the amount of resistance in the discharge circuits of the various cylinders, the curves could be drawn to scale to imitate approximately the variation in amount of the various products with time when the initial conditions are given.

During the last few years a very large amount of work has been done in tracing the remarkable succession of transformations that occur in the various radio-active substances. The known products of radium, thorium, actinium, and uranium are shown graphically below, together with the periods of the products and the character of the radiations they emit. It will be seen that a large list of these unstable bodies are now known. It is probable, however, that not many more remain to be discovered. The main uncertainty lies in the possibility of overlooking a product of rapid transformation following or succeeding one with a very slow period. In tracing out the succession of changes, the emanations or radio-active gases continuously evolved by radium, thorium, and actinium have marked a very definite and important stage, for these emanations can be easily removed from the radio-active body and their further transformations studied quite apart from the parent element. The analysis of the transformation of the radium emanation has yielded results of great importance and interest. After passing through three stages, radium $\mathrm{A}, \mathrm{B}$, and $\mathrm{C}$, of short period, a substance, radium $\mathrm{D}$, of long period, makes its appearance. This is transformed through two stages $E$ and $F$ of short period into radium G, of period 140 days. Meyer and Schweidler have conclusively shown that radium $D$ is the primary 
constituent of the radio-active substance separated by Hofmann and called by him radio-lead. Radium $G$ is identical with the first radio-active substance separated from pitchblende by Madame Curie, viz. polonium. We are thus sure that these bodies are transformation products of radium. It will be seen that $\mathrm{I}$ have added another product of period 4.5 days between radium $\mathrm{D}$ and polonium. The presence of such a product has been shown by Meyer and Schweidler.

In the case of thorium, a very long list of products is now known. For several years thorium $\mathrm{X}$ was thought to be the first product of thorium, but $\mathrm{Hahn}$ has recently shown that at least two other products of slow transformation intervene, which he has called mesothorium and radiothorium. The radiothorium emits $a$ rays, and has a period of more than 800 days. Mesothorium apparently emits $\beta$ rays, and has a still longer period of transformation, the exact value of which has not yet been accurately determined. Since thorium is used commercially on a large scale, there is every prospect that we shall soon be able to obtain considerable quantities of very active preparations of mesothorium and radiothorium. The separation of these bodies from thorium does not in any way alter its commercial value. It is to be hoped that if these active preparations are separated in quantity, the physicist and chemist may be able to obtain a supply of very active material at a reasonable cost, and that there will not be an attempt to compete with the ridiculously high prices charged for radium.

From the radio-active point of view, the radio-elements are only distinguished from their families of products by their comparatively long period of transformation. Noty we have reason to believe that radium itself is transformed according to the laws of other radio-active products with a period of about 2000 years. If this be the case, in order to keep up its supply in a mineral, radium must be produced from another substance of relatively long period of transformation. The search for this elusive parent of radium has been one of almost dramatic interest, and illustrates the great importance of the theory as a guide to the experimenter. The view that radium was a substance in continuous transformation was put forward by Rutherford and Soddy in

1903. The most probable parent of radium appeared to be uranium, which has a period of transformation of the order of 1000 million years. If this were the case, uranium, initially freed from radium, should in the course of time grow radium, i.e. radium should again appear in the uranium. This has been tested independently by Soddy and Boltwood, and both have shown that in carefully prepared uranium solutions there is no appreciable growth of radium in the course of several years. The rate of production of radium, if it occurs at all, is certainly less than $\mathrm{I} /$ rooo of the amount to be expected from theory. This would appear at first sight to put out of count the view that uranium is the parent of radium. This, however, is by no means the case, for such a result could be very easily explained if one or more substances of very slow period of transformation appeared between uranium and radium. It is obvious that the necessity of forming such an intermediate product would greatly lengthen the time required before an appreciable amount of radium appeared.

There is, however, another indirect but very simple method of attack to settle the parentage of radium. If radium is derived from the transformation of uranium, however many unknown products intervene, the ratio between the amount of radium and uranium in old minerals should be a definite constant. This is obviously the case, provided sufficient time has elapsed for the amount of radium to have reached its equilibrium value. The constancy of this relation has been completely substantiated by the independent work of Boltwood, Strutt, and McCoy. It has been shown that the quantity of radium corresponding to I gram of radium is $3.8 \times \mathrm{xo}^{-7} \mathrm{gram}$, and is the same for minerals obtained from all parts of the world. Since the radium is always distributed throughout the mass of uranium, we cannot expect to find nuggets of radium like nuggets of gold, unless by some chance the radium has been dissolved out of radio-active minerals and redeposited within the last few thousands of years. To those who had faith in the disintegration theory, this unique constant relation between the amounts of two elements was a satisfactory proof that radium stood in a genetic relation with uranium. A search was then made for the unknown intervening product which, if isolated, must grow radium at a rapid rate. A year or so ago Boltwood observed that a preparation of actinium separated
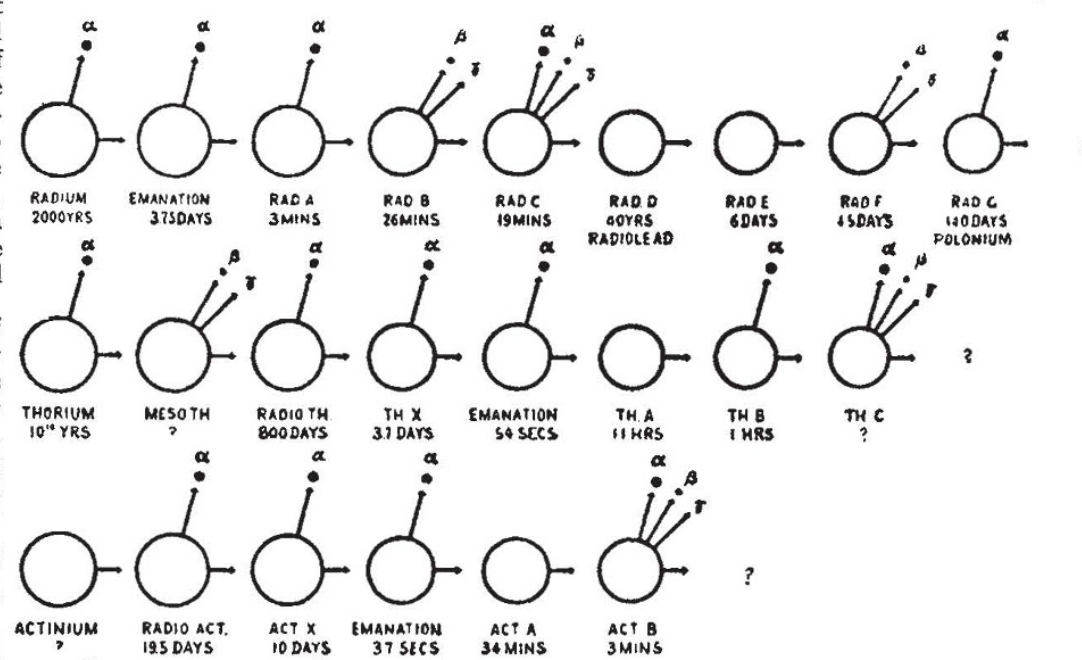
NO. 2001 , vOL. 77$]$ 
more than one thousand million years, it will not be profitable at the moment to try and trace back the family further.

It appears almost certain that, from the radio-active point of view, uranium and thorium must be considered as two independent elements. The case of actinium is different, for Boltwood has shown that the amount of actinium in minerals, like the amount of radium, is proportional to the amount of uranium. This indicates that actinium stands in a genetic relation with uranium. Unless our experimental evidence is at fault, it does not appear probable that actinium belongs to the main line of descent of uranium, for the activity of actinium separated from a mineral compared with radium is only about onequarter of what we should expect under such conditions. I think that a suggestion which I put forward some time ago may account for the obvious connection of actinium with uranium, and at the same time for the anomaly observed. This supposes that actinium is a branch descent from some member of the uranium family. It does not appear improbable that at one stage of the disintegration two distinct substances may be produced, one in greater quantity than the other. After the expulsion of an a particle, it may happen that there are two possible arrangements of temporary stability of the residual atom. The great majority of the atoms may fall into one arrangement, and the remainder into the other. Actinium in this case would correspond to the substance in lesser quantity. It would act as a distinct element, and would break up in a different way from the main amount. It is probable that a large amount of accurate work will be required before the position of actinium in the scheme of changes can be fixed with certainty. It is a matter of remark how closely actinium resembles thorium in its series of transformations. It would appear that the atom of actinium has many points in common with thorium, or rather with its product, mesothorium.

The recent observations on the growth of radium offer a very simple and straightforward method of determining experimentally the period of radium. Suppose that we take a uranium mineral and determine by the emanation method the quantity of radium contained in it. If the immediate parent of radium (i.e. ionium) is next completely separated from the uranium and radium, it will begin to grow radium at a constant rate. Now the rate of growth of radium observed is a measure of the rate of breaking up of the radium parent in the mineral, since before separation the rate of production was equal to the rate of breaking up. Now the growth of radium observed for short interval, for example, a year, divided by the quantity present in the mineral, gives the fraction of the radium breaking up per year. Proceeding in this way, Boltwood found that the fraction breaking up per year is about $1 / 3000$, and that the period of radium is about 2000 years --a value which lies between the most probable values deduced from quite distinct data.

From an inspection of the radio-active families, it will be seen that out of twenty-six radio-active substances that have been identified, seventeen give out $\alpha$ rays or $\alpha$ and $\beta$ rays, four give out only $\beta$ rays, and five emit no rays at all. The rayless and $\beta$-ray products are transformed according to the same law as the a-ray products, and there is the same sudden change of physical and chemical properties as the result of the transformation. In the case of the substances which throw off atoms of matter in the form of a particles, there are obvious reasons for anticipating a change in properties of the substance, but this is not the case for the rayless or $\beta$-ray products. We must either suppose that the mass of the atom is not appreciably changed by the transformation, which consists in an internal rearrangement of the parts of the atom, or that the atom expels a particle at too low a velocity to be appreciated by the electrical methods. Unfortunately, it is very difficult to study the rayless products with care, as in practically every case they are succeeded by a ray product of comparatively rapid transformation. The ravless products are of great interest as indicating the possibility of transformations which can occur without any detectable radiation.

In the course of the analysis of radio-active clianges special methods have been developed for the separalion of the various products from each other. It is only in a few cases, however, that we can hope to obtain a sufficient quantity of the substance to examine by means of the balance. It should be possible to obtain workable quantities of actinium, radium $D$ (radio-lead), and radium $G$ (polonium), but the isolation of these substances in any quantity has not yet been effected. Sir William Ramsay and Mr. Cameron have made a number of important investigations of the properties and volume of the radium emanation, freed so far as possible from any traces of known gases. The remarkable initial contraction of the volume due to the emanation shows that there is still much to be done to obtain a clear understanding of the behaviour of this intensely radio-active gas when obtained in a pure state.

Simultaneously with the work on the analysis of radioactive changes, a large number of investigations have been made on the laws of absorption by matter of the three primary types of radiation from active matter, viz. the $\alpha, \beta$, and $\gamma$ rays, and the secondary radiations to which they give rise. It has generally been accepted for some years that the $\gamma$ rays are a type of penetrating X-rays. The latter are supposed to consist of electromagnetic pulses in the ether, set up by the impact or escape of electrons from matter, and akin in many respects to very short waves of ultra-violet light. Recently, however, Bragg has challenged this view, and has suggested that the $\gamma$ rays (and probably also the X-rays) are mainly corpuscular in character, and consist of uncharged particles or "neutral pairs," as he terms them, projected at a high velocity. Such a view serves to explain most of the experimental observations equally well as the pulse theory; Bragg has recently brought forward additional evidence, based on the direction of the secondary radiation from the $\gamma$ rays, which he considers to be inexplicable by the pulse theory. We must await further data before this important question can be settled definitely, but the theory of Bragg, which carries many important consequences in its train, certainly deserves very careful examination.

From the radio-active point of view, the $\alpha$ rays are by far the most important type of radiation emitted by active matter, although their power of penetration is insignificant compared with the $\beta$ or $\gamma$ rays. They consist of veritable atoms of matter projected at a speed, on an average, of 6000 miles per second. It is the great energy of motion of these swiftly expelled masses that gives rise to the heating effect of radium. In addition, they are responsible for the greater part of the ionisation observed near an uncovered radio-active substance. On account of their importance in radio-active phenomena, I shall devote some little attention to the behaviour of these rays. The work of Bragg and Kleeman, of Adelaide, first gave us a clear idea of the nature of the absorption of these rays by matter. The $\alpha$ particles from a very thin film of any simple kind of radio-active matter are all projected at an identical speed, and lose their power of ionising the gas or of producing phosphorescence or photographic action after they have traversed exactly the same distance, which may conveniently be called the "range" of the a particle. Now every product emits $\alpha$ particles at an identical speed among themselves, but different from every other product. For example, the swiftest a particles from the radium family, viz. that from radium $\mathrm{C}$, travels $7 \mathrm{~cm}$. in air under ordinary conditions before it is stopped, while that from radium itself is projected at a slower speed, travelling only $3.5 \mathrm{~cm}$. We may regard the $\alpha$ particle as a projectile travelling so swiftly that it plunges through every molecule in its path, producing positively and negatively charged jons in the process. On an average, an $\alpha$ particle before its career of violence is stopped breaks up about Ioo, ooo molecules. So great is the kinetic energy of the a projectile that its collisions with matter do not sensibly deflect it, and in this respect it differs markedly from the $\beta$ particle, which is apparently easily deflected by its passage through matter. At the same time, there is undoubted evidence that the direction of motion of some of the $\alpha$ particles is slightly changed by their passage through matter.

The sudden cessation of the ionising power produced by the a particle after traversing a definite distance of air has bcen shown by Bragg to be a powerful method of 
analysis of the number of $\alpha$-ray products present in a substance. For example, suppose the amount of ionisation in the gas produced by a narrow pencil of a rays is examined at varying distances from the radium. At a distance of $7 \mathrm{~cm}$. there is a sudden increase in the amount of ionisation, for at this distance the a particles from radium $\mathrm{C}$ enter the testing vessel. There are again sudden changes in the ionisation at distances of $4.8 \mathrm{~cm} ., 4.3 \mathrm{~cm}$., and $3.5 \mathrm{~cm}$. These are due to the rays from the radium $\mathrm{A}$, the emanation and radium itself respectively entering the testing vessel. The $\alpha$-ray analysis thus discloses four types of a rays present in radium in equilibrium-a result in conformity with the more direct analysis. This method allows us to settle at once whether more than one a-ray product is present in a given radio-active material. For example, an analysis by Hahn by this method of the radiation from the active deposit of thorium has disclosed the existence of two a-ray products instead of one as previously supposed. We can consequently gain information on the complexity of radio-active material, even though no chemical methods have been found to separate the products concerned. The range of the $\alpha$ particle from each product is a definite constant which is characteristic of each product.

The a particle decreases in velocity as it passes through matter. This result is clearly brought out by photographs showing the deflection of a homogeneous pencil of $a$ rays in a magnetic field before and after passing through an absorbing screen. The greater divergence of the trace of the a rays on the plate, after passing through the screen, shows that their velocity is reduced, while the sharpness of the band shows that the $\alpha$ particles still move at an identical speed.

In order to make an accurate determination of the constants of the a particles, it is necessary to work with homogeneous rays, and we consequently require to use a thin layer of matter of one kind. For experiments of this character, a wire coated with a thin film of radium $\mathrm{C}$ by exposure to the radium emanation is very suitable. The velocity of the $\alpha$ particle and the value $\mathrm{e} / \mathrm{m}$, the ratio of the charge carried by the $\alpha$ particle to its mass, can be deduced by observing the deflections of a pencil of a rays exposed in a magnetic and in an electric field of known strengths. The deflection of a pencil of a rays in an electric field is small under normal conditions, and special care is needed to determine it with accuracy.

In this way I have calculated the velocity and value of $\mathrm{e} / \mathrm{m}$ for a number of $\alpha$-ray products. The velocity of expulsion varies for different products, but is connected by a simple relation with the range of the $\alpha$ particle in air. The value of $\mathrm{e} / \mathrm{m}$ has been determined for selected products of radium, thorium, and actinium, and in each case the same value has been found. This shows that the a particles expelled from radio-active substances in general are identical in constitution. They have all the same mass, but differ from one another in the initial velocity of their projection. Although we are sure that the $\alpha$ particles, from whatever source, are identical atoms of matter, we are still unable to settle definitely the true nature of the $a$ particle. The value of $\mathrm{e} / \mathrm{m}$ found by experiment is nearly $5 \times 10^{3}$. Now the value of $\mathrm{e} / \mathrm{m}$ for the hydrogen atom in the electrolysis of water is $10^{4}$. If the charge carried by the $\alpha$ particle and the hydrogen atom is the same, the mass of the $\alpha$ particle is twice that of the hydrogen atom, i.e. a mass equal to the hydrogen molecule. But we are not certain that they do carry the same charge. Here we are, unfortunately, confronted by a number of possibilities, for the magnitude of $m$ for the $\alpha$ particle is conditioned by the value assumed for $e$. If the charge of the $\alpha$ particle is assumed to be twice the value of the hydrogen atom, the mass comes out four times the hydrogen atom-the value found for the helium atom. The weight of evidence still supports the view that the $\alpha$ particle is in some way connected with the helium atom. If the a particle is a helium atom with twice the ionic charge, we must regard the helium produced by radio-active bodies as actually the collected $\alpha$ particles the charges of which have been neutralised. This at once offers a reasonable explanation of the production of helium by actinium as well as by radium. In addition, Strutt has recently contributed strong evidence that helium is a product of thorium. Such results are only to be expected on the above view, since the $\alpha$ particle is the only common product of these elements.

The determination of the true character of the $\alpha$ particle is one of the most pressing unsolved problems in radioactivity, for a number of important consequences follow from its solution. Unfortunately, a direct experimental proof of its true character appears to be very difficult unless a new method of attack is found. We have seen that if the charge carried by the $\alpha$ particle could be experimentally determined, the actual value of $m$ could be determined in terms of the hydrogen atom, since the value of the charge carried by the latter is known. This could be done if we could devise a method of detecting the emission of a single $a$ particle, and thus counting the number of particles expelled from a known quantity of a radio-active substance, for example, from radium. In considering a possible method of attack of this question, the remarkable property of the a particles of producing scintillations in zinc sulphide at once suggests itsclf. Apart from the difficulty of counting the scintillations, it is very doubtful whether more than a small fraction of the $\alpha$ particles which strike the screen produce the scintillations. Viewed from the electrical side, a simple calculation from the data at our disposal shows that the ionisation produced in a gas by a single a particle should be detectable. The electrometer or electroscope used for measurement would, however, require to be extremely sensitive, and under such conditions it is known that small electrical disturbances are very difficult to avoid.

In order to obtain a reasonably large effect, we require some method of magnifying the ionisation produced by the $\alpha$ particle. In conjunction with Dr. Hans Geiger, I have recently developed a method whereby the electrical effect produced by the a particle can be magnified several thousand times. From the work of Townsend it is known that if a strong electric field acts on gas at low pressure, any ions generated in the gas by an external agency are set in motion by the electric field, and under the proper conditions produce fresh ions by collision with the gas molecules. The negative ion is the most effective ioniser in weak fields, but when the voltage is increased near the point at which a discharge passes, the positive ion also produces fresh ions by collision. In the experimental arrangement the $\alpha$ particle from the active matter is fired through a small opening about $2 \mathrm{~mm}$. in diameter, covered with a thin layer of mica, into a cylinder $60 \mathrm{~cm}$. Iong and $2.5 \mathrm{~cm}$. in diameter, in which the gas pressure is about $3 \mathrm{~cm}$. of mercury. A thin insulated wire connected to the electrometer is fixed centrally in the cylinder. If the outside cylinder is charged negatively, for a difference of potential of about 1000 volts. any ionisation produced in the cylinder is increased about 2000 times by collision. This can be simply illustrated by using the $\gamma$ rays of radium as a source of jonisation. When a difference of potential is applied to the cylinder, the ionisation produced by the $\gamma$ rays only causes a slight movement of the electrometer needle. By applying, however, a voltage ncarly equal to that required for a discharge through the gas there is a very rapid movement of the needle. On removing the radium there is no appreciable current through the gas. On placing a source of $\alpha$ rays near the small opening in the cylinder so that some of the $\alpha$ particles can be fired along the axis of the cylinder, the electrometer needle does not move uniformly, but with a succession of rapid throws with a considerable interval in between. Each of these throws is due to the discharge produced by a single $\alpha$ particle entering the cylinder, increased several thousand times by the intermediary of the strong electric field. If a sheet of paper which stops the $\alpha$ rays is placed before the opening, the electrometer needle at once comes to rest. The interval of time between the throws is not uniform. This is exactly what we should expect if the number of $\alpha$ particles entering such a small opening is governed by the law of probability. On the average, a certain number of $\alpha$ particles are fired through the opening per minute, but in some cases the interval is less than the average, in others much greater. In fact, by observing the intervais between the entrance of a large number of a particles, we should be able to determine accurately the "probability" curve of distribu- 
tion of the a particles with time. For purposes of measurements, the active material, in the form of a thin film covering a small area, is placed in an exhausted tube connected in series with the ionisation cylinder, and at a considerable distance from the hole. The number of $\alpha$ particles entering the opening per minute is counted, and from this the total number expelled can be calculated. Preliminary measurements show that the number of a particles expelled from a known weight of radium is of the same order as the calculated value. When the measurements are completed it should be possible to determine the charge carried by each $\alpha$ particle, since the total charge carried by the $\alpha$ particles from I gram of radium is known. In this way it may be possible to settle whether the a particle is a helium atom or not. In any case, it is a matter of some interest to be able to detect by its electrical effect a single atom of matter, and so to determine directly with a minimum of assumption the magnitude of some of the most important quantities in radio-active phenomena.

\section{MEDICAL INSPECTION OF SCHOOL CHILDREN. ${ }^{1}$}

THE memorandum issued by the English Board of Education on the medical inspection of children in public elementary schools is a statesmanlike document. It propounds a policy; it indicates a method, and the method, no less than the policy, takes full account of conditions, difficulties, and obstacles. The memorandum gives body to the provisions of section 3 of the Education (Administrative Provisions) Act, 1907. This section confers three broad powers on education authorities, first, to provide special environments for special children, e.g. vacation schools, vacation classes, play centres, \&c.; second, to establish a medical inspection of the individual children; thirdly, "to make such arrangements as may be sanctioned by the Board of Education for attending to the health and physical condition of the children educated in public elementary schools." These three powers may be exercised in cooperation with voluntary agencies, of which, it is needless to say, there are many. But the point of importance is that the powers may now be exercised by the education authorities, and practically, since grants may be made to depend on their exercise, the education authorities are now placed under obligation to carry them into full effect. The memorandum proceeds on this assumption; but it aims rather at sketching a process of natural administrative growth than at imposing an imperative system to be immediately realised. Accordingly, it starts from what is already being done in several localities to supervise the hygiene of schools and scholars. The sanitary authorities are in possession. This Act does not supersede, it expands and supplements, their work. Here emerges the cardinal principle of the memorandum, namely, the extension of the conception of public health to include, not merely the environmental sanitation considered apart, but the individual child's health as it is affected by his environment in the widest sense-physical, educational, \&c.

The purpose of individual inspection, no less than of the general inspection of the hygienic conditions, is " to secure ultimately for every child, normal or defective, conditions of life compatible with that full and effective development of its organic functions, its special senses and its mental powers which constitute a true education." Unfortunately, owing to accidents of administrative convenience or development, there has arisen within the medical profession an acute difference of opinion as to the relative advantages of a special school medical service and an expanded public health service. Dr. Newman's appointment implied that the Board of Education favoured the idea of an expanded public health service, and this memorandum sketches in firm outline what this view implies. Incident-

1 ( $)$ Memorandum on Medical Inspection of Children in Public Elementary Schools, under Section 13 of the Education (Administrative Provisions) Act. I907 (Bnard of Education ; Circular, 576).

(2) Memorandum by British Medical Association on the Circular of the Board of Education (British Medical Journal, Supplement, December 21, I907.)

(3) Schedule of Medical Inspection (accompanying Circular 582) NO. $200 \mathrm{I}$, VOL. 77$]$ ally, it shows that there is no opposition between the two views. On the one hand, it puts upon the medical officer of health the organising of the system of medical inspection, but on the other, it provides that "its actual execution" shall be "deputed wholly or partly to suitable colleagues or assistants (men or women)." The two factions are thus reconciled in the one administrative organisation.

The memorandum in more than one place emphasises in a way that it is impossible to controvert the primary importance of the home and its hygiene in the school-life of the child, and the absolute necessity for maintaining continuity of inspectorial interest between the home and the school. Medical inspection will thus work backwards to the home and forwards to the after-school life of the child, so covering the entire period between birth and the entry on industrial life. When this conception of continuity is fully grasped, there will be no further theoretical dispute between the medical factions concerned.

The British Medical Association has issued a memorandum dealing in a thoroughly practical spirit with the proposals and suggestions of the Board of Education. It is of immense importance that the medical profession should thus declare itself at the beginning. The differences between the association and the Board are essentially differences of detail. The association is quite frank in its acceptance of the general positions. The association's memorandum states that " these duties could not, having regard to the nature and extent of the duties already required of Medical Officers of Health, be efficiently discharged by them personally." This is not inconsistent with the Board's suggestions on the same point. The association also states that "part-time" medical officers, paid as for work done, could appropriately 'undertake medical inspection. This comes naturally from the profession, and there is much to say for it; but again there is nothing here inconsistent with the Board's views. But just as in the earlier, so in these later expansions of preventive medicine, the tendency will be towards "wholetime" specialists. In England many of the counties have not yet appointed whole-time or even part-time medical officers-so differing from Scotland, where every county is obliged to appoint a medical officer, and all except five have appointed whole-time men.

The association's memorandum is emphatic on another point, namely, that treatment of disease and visitation of the homes of the children shall be excluded from the scope of the medical inspector's duties. The full bearing of this suggestion will require very careful consideration. The Education Board's memorandum contains a very judicious discussion of the implications of the Act as to treatment, and it is difficult to reconcile the Act with the letter of the association's decision. The schedule proposed by the association is very well drawn, but it makes no provision for any record as to home conditions or occupation of parents, \&c., which are insisted on in the Board's memorandum.

The Board of Education has followed up its memorandum by a detailed schedule, with full directions for the medical inspection. In most respects; this schedule meets all the proposals of the British Medical Association. From the tenor of the memorandum on the clear necessity for recording the home conditions and the occupational condition of the parents, we naturally expected that these points would be explicitly provided for in the schedule. In this we are somewhat disappointed; for all that we find is a heading for "Directions to Parent or Teacher." It would have been much simpler to have specified what details are wanted for every child-number of rooms in house, number of persons, occupation of father or mother, preand post-school labour of the child. These are all primary factors in the mental state of the child at any one time, and practically all these data are already in possession o the school authority. In other respects, the schedule is very comprehensive. Indeed, this is the one real criticism offered by medical critics. But when it is closely scrutinised, it will be found to contain only the bare essentials of a real inspection. The order of the schedulc is simple, and the directive notes are models of lucidity. The anthropologist may regret that his special point of view is not as such provided for, but there is nothing 